\title{
ISOMORPHISM CLASSES OF ELLIPTIC CURVES OVER A FINITE FIELD IN SOME THIN FAMILIES
}

\author{
Javier Cilleruelo, Igor E. Shparlinski And Ana Zumalacárregui
}

\begin{abstract}
For a prime $p$ and a given square box, $\mathfrak{B}$, we consider all elliptic curves $E_{r, s}: Y^{2}=X^{3}+r X+s$ defined over a field $\mathbb{F}_{p}$ of $p$ elements with coefficients $(r, s) \in \mathfrak{B}$. We obtain a nontrivial upper bound for the number of such curves which are isomorphic to a given one over $\mathbb{F}_{p}$, in terms of the size of $\mathfrak{B}$. We also give an optimal lower bound on the number of distinct isomorphic classes represented.
\end{abstract}

\section{Background and notation}

For a prime $p$ we consider the family of elliptic curves $E_{a, b}$ given by a Weierstrass equation

$$
E_{a, b}: \quad Y^{2}=X^{3}+a X+b
$$

over the finite field $\mathbb{F}_{p}$ of $p$ elements, where

$$
(a, b) \in \mathbb{F}_{p}^{2}, \quad 4 a^{3}+27 b^{2} \neq 0 .
$$

Recall that for a large enough prime, say $p>3$, it is well known that every elliptic curve over $\mathbb{F}_{p}$ has a representation of this type, see [13] for a background on elliptic curves. Thus, from now on, curves are considered as parameterized by their coefficients.

Two curves $E_{r, s}$ and $E_{u, v}$ are isomorphic if for some $t \in \mathbb{F}_{p}^{*}$ we have

$$
r t^{4} \equiv u \quad(\bmod p) \quad \text { and } \quad s t^{6} \equiv v \quad(\bmod p)
$$

There are several works which count the number of curves $E_{r, s}$ isomorphic to a given curve $E_{a, b}$, with coefficients $r, s$ lying in certain box $(r, s) \in[R+1, R+K] \times$ $[S+1, S+L]$, see $[2,8]$. In particular, for

$$
K L \geq p^{3 / 2+\varepsilon} \quad \text { and } \quad \min \{K, L\} \geq p^{1 / 2+\varepsilon}
$$

with some fixed $\varepsilon>0$, using exponential sum techniques, Fouvry and Murty [8] have obtained an asymptotic formula for every pair $(a, b)$ with (1.1). In [2], using bounds of multiplicative character sums, for almost all $(a, b)$ with (1.1), this condition (1.3) has been relaxed to

$$
K L \geq p^{1+\varepsilon} \quad \text { and } \quad \min \{K, L\} \geq p^{1 / 4+\varepsilon} .
$$

Furthermore, it is shown in [2], that for

$$
K L \geq p^{1+\varepsilon} \quad \text { and } \quad \min \{K, L\} \geq p^{1 / 4 e^{1 / 2}+\varepsilon},
$$

Received by the editors June 2, 2011. 
one can get a lower bound with the right order of magnitude (again for almost all $(a, b)$ with (1.1)). On average over $p$, such results are established for even smaller boxes, see [2].

Here we consider squared boxes, much smaller than the previous ones, given by

$$
\mathfrak{B}=[R+1, R+M] \times[S+1, S+M] \subseteq \mathbb{F}_{p} \times \mathbb{F}_{p},
$$

for a prime $p$ and some nonnegative integers $R, S, M$ satisfying

$$
R, S \geq 0, M \geq 1 \quad \text { and } \quad R+M, S+M<p .
$$

We use $|\mathfrak{B}|$ to denote the area of $\mathfrak{B}$, that is,

$$
|\mathfrak{B}|=M^{2} \text {. }
$$

We are interested in understanding how isomorphism classes are distributed in such small boxes $\mathfrak{B}$. Among all curves $E_{r, s}$, parameterized by coefficients $(r, s) \in \mathfrak{B}$, we study, in first place, the number of isomorphism classes which are represented and, finally, the number of curves lying in a given isomorphism class.

Clearly, the existence of an isomorphism between $E_{r, s}$ and $E_{u, v}$, see (1.2), implies that

$$
r^{3} v^{2} \equiv u^{3} s^{2} \quad(\bmod p)
$$

We denote by $T(\mathfrak{B})$ the number of solutions to (1.6) with $(r, s),(u, v) \in \mathfrak{B}$. Furthermore, for $\lambda \in \mathbb{F}_{p}$, we denote by $N_{\lambda}(\mathfrak{B})$ the number of solutions to the congruence

$$
r^{3} \equiv \lambda s^{2} \quad(\bmod p), \quad(r, s) \in \mathfrak{B} .
$$

We use the bounds of character sums detailed in Section 2 to obtain an upper bound on $T(\mathfrak{B})$. From this estimate we derive an almost optimal lower bound for the number $I(\mathfrak{B})$, of nonisomorphic curves with coefficients in $\mathfrak{B}$, of the form

$$
I(\mathfrak{B}) \geq \min \left\{(1+o(1)) p,|\mathfrak{B}|^{1+o(1)}\right\}
$$

see Corollary 4.1 below for a more precise formulation.

Clearly, the bound (1.7) is quite tight as we have the trivial upper bound

$$
I(\mathfrak{B}) \leq \min \{2 p+O(1),|\mathfrak{B}|\},
$$

since it is well known [12] that the number of isomorphism classes of elliptic curves in $\mathbb{F}_{p}$ is $2 p+O(1)$.

Finally, we exploit the method of [5], based on the ideas of [4] (see also [15]), to obtain in Section 5 upper bounds on $N_{\lambda}(\mathfrak{B})$, which, in particular, imply upper bounds for the number of elliptic curves $E_{r, s}$ with coefficients $(r, s) \in \mathfrak{B}$ that fall in the same isomorphism class.

Throughout the paper, any implied constants in the symbols $O, \ll$ and $\gg$ are absolute. We recall that the notations $U=O(V), U \ll V$ and $V \gg U$ are all equivalent to the statement that the inequality $|U| \leq c V$ holds with some constant $c>0$. Furthermore the notation $U=V^{o(1)}$ is equivalent to the statement that for every $\varepsilon>0$ the inequality $U \leq c(\varepsilon) V^{\varepsilon}$ holds for some constant $c(\varepsilon)>0$ that depends only on $\varepsilon$. 


\section{Character sums}

Let $\mathcal{X}$ be the set of all multiplicative characters modulo $p$ and let $\mathcal{X}^{*}=\mathcal{X} \backslash\left\{\chi_{0}\right\}$ be the set of nonprincipal characters.

We recall the Pólya-Vinogradov bound, see [11, Theorem 12.5].

Lemma 2.1. For arbitrary integers $W$ and $Z$, with $0 \leq W<W+Z<p$, the bound

$$
\max _{\chi \in \mathcal{X}^{*}}\left|\sum_{z=W+1}^{W+Z} \chi(z)\right| \ll p^{1 / 2} \log p
$$

holds.

We recall that Garaev and García [9], improving a result of Ayyad et al. [1] (see also [6]), have shown that for any integers $W$ and $Z$

$$
\sum_{\chi \in \mathcal{X}^{*}}\left|\sum_{z=W+1}^{W+Z} \chi(z)\right|^{4} \ll p Z^{2}\left(\log p+\left(\log \left(Z^{2} / p\right)\right)^{2}\right) .
$$

Note that for any fixed $\varepsilon>0$, if $Z \geq p^{\varepsilon}$ the right-hand side of (2.1) is of the form $p Z^{2+o(1)}$. However for small values of $Z$, namely for $Z \ll(\log p)^{1 / 2}$, the bound $(2.1)$ is trivial. We now combine (2.1) with a result of [4] to get the bound $p Z^{2+o(1)}$ for any $Z$.

Lemma 2.2. For arbitrary integers $W$ and $Z$, with $0 \leq W<W+Z<p$, the bound

$$
\sum_{\chi \in \mathcal{X}^{*}}\left|\sum_{z=W+1}^{W+Z} \chi(z)\right|^{4} \ll p Z^{2+o(1)}
$$

holds.

Proof. We can assume that $Z \leq p^{1 / 4}$ since otherwise, as we have noticed before, the bound (2.1) implies the desired result. Now, using that for any integer $z$ with $\operatorname{gcd}(z, p)=1$, for the complex conjugated character $\bar{\chi}$ we have

$$
\bar{\chi}(z)=\chi\left(z^{-1}\right)
$$

we derive,

$$
\sum_{\chi \in \mathcal{X}^{*}}\left|\sum_{z=W+1}^{W+Z} \chi(z)\right|^{4} \leq \sum_{\chi \in \mathcal{X}}\left|\sum_{z=W+1}^{W+Z} \chi(z)\right|^{4}=\sum_{z_{1}, z_{2}, z_{3}, z_{4}=W+1}^{W+Z} \sum_{\chi \in \mathcal{X}} \chi\left(z_{1} z_{2} z_{3}^{-1} z_{4}^{-1}\right) .
$$

Thus, using the orthogonality of characters we obtain

$$
\sum_{\chi \in \mathcal{X}^{*}}\left|\sum_{z=W+1}^{W+Z} \chi(z)\right|^{4} \leq p J
$$

where $J$ is number of solutions to the congruence

$$
z_{1} z_{2} \equiv z_{3} z_{4} \quad(\bmod p), \quad z_{1}, z_{2}, z_{3}, z_{4} \in[W+1, W+Z]
$$

By $[4$, Theorem 1$]$, for any $\lambda \not \equiv 0(\bmod p)$ the congruence

$$
z_{1} z_{2} \equiv \lambda \quad(\bmod p), \quad z_{1}, z_{2} \in[W+1, W+Z]
$$


has $Z^{o(1)}$ solutions, provided that $Z \leq p^{1 / 4}$. Therefore $J \leq Z^{2+o(1)}$ and the result follows.

\section{Small points on some hypersurfaces}

For the number of points in very small boxes we can get a better bound by using the following estimate of Bombieri and Pila [3] on the number of integral points on polynomial curves.

Lemma 3.1. Let $\mathcal{C}$ be an absolutely irreducible curve of degree $d \geq 2$ and $H \geq$ $\exp \left(d^{6}\right)$. Then the number of integral points on $\mathcal{C}$ and inside of a square $[0, H] \times[0, H]$ does not exceed $H^{1 / d} \exp (12 \sqrt{d \log H \log \log H})$.

For an integer $a$ we used $\|a\|_{p}$ to denote the smallest by absolute value residue of $a$ modulo $p$, that is

$$
\|a\|_{p}=\min _{k \in \mathbb{Z}}|a-k p|
$$

By the Dirichlet pigeon-hole principle we easily obtain the following result.

Lemma 3.2. For any real numbers $T_{1}, \ldots, T_{s}$ with

$$
p>T_{1}, \ldots, T_{s} \geq 1 \quad \text { and } \quad T_{1} \cdots T_{s}>p^{s-1}
$$

and any integers $a_{1}, \ldots, a_{s}$ there exists an integer $t$ with $\operatorname{gcd}(t, p)=1$ satisfying

$$
\left\|a_{i} t\right\|_{p} \ll T_{i}, \quad i=1, \ldots, s .
$$

\section{Bound on $T(\mathfrak{B})$}

In fact we consider a more general quantity, that is for given positive integers $i, j$ we bound the number $T_{i, j}(\mathfrak{B})$ of solutions to the equation

$$
r^{i} v^{j} \equiv u^{i} s^{j} \quad(\bmod p)
$$

with $(r, s),(u, v) \in \mathfrak{B}$. Thus, in this setting, $T(\mathfrak{B})=T_{3,2}(\mathfrak{B})$.

Theorem 4.1. For any prime $p$ and any box $\mathfrak{B}$ given by (1.4) and satisfying (1.5) we have,

$$
T_{i, j}(\mathfrak{B})=d \frac{|\mathfrak{B}|^{2}}{p-1}+O\left(|\mathfrak{B}| p^{o(1)}\right)
$$

as $|\mathfrak{B}| \rightarrow \infty$, where $d=\operatorname{gcd}(i, j, p-1)$.

Proof. Using the orthogonality of characters, we write the number of solutions to (4.1) with $(r, s),(u, v) \in \mathfrak{B}$ as

$$
\begin{aligned}
T_{i, j}(\mathfrak{B}) & =\sum_{r, u=R+1}^{R+M} \sum_{s, v=S+1}^{R+M} \frac{1}{p-1} \sum_{\chi \in \mathcal{X}} \chi\left((r / u)^{i}(v / s)^{j}\right) \\
& =\frac{1}{p-1} \sum_{\chi \in \mathcal{X}}\left|\sum_{r=R+1}^{R+M} \chi^{i}(r)\right|^{2}\left|\sum_{s=S+1}^{S+M} \chi^{j}(s)\right|^{2} .
\end{aligned}
$$

The contribution to the above sum from $d$ characters $\chi \in \mathcal{X}$ with $\chi^{i}=\chi^{j}=\chi_{0}$ is $d M^{4} /(p-1)$. 
Using Lemma 2.1, we see that the contribution to the above sum from at most $i$ characters $\chi \in \mathcal{X}$ with $\chi^{i}=\chi_{0}$ and $\chi^{j} \neq \chi_{0}$ is bounded by

$$
\frac{M^{2}}{p-1} \sum_{\substack{\chi \in \mathcal{X} \\ \chi^{i}=\chi_{0}}}\left|\sum_{s=S+1}^{S+M} \chi^{j}(s)\right|^{2} \ll M^{2}(\log p)^{2} .
$$

The contribution from the characters $\chi \in \mathcal{X}$ with $\chi^{j}=\chi_{0}$ and $\chi^{i} \neq \chi_{0}$ can be estimated similarly as $O\left(M^{2} \log p\right)$.

Therefore

$$
T_{i, j}(R, S ; M)=d \frac{M^{4}}{p-1}+O\left(M^{2}(\log p)^{2}+W\right),
$$

where

$$
W=\left.\frac{1}{(p-1)^{2}} \sum_{\substack{\chi \in \mathcal{X} \\ \chi^{i}, \chi^{j} \neq \chi_{0}}}\left|\sum_{r=R+1}^{R+M} \chi^{i}(r)\right|^{2}|| \sum_{s=S+1}^{S+M} \chi^{j}(s)\right|^{2} .
$$

Using the Cauchy inequality, we derive

$$
W^{2} \leq \frac{1}{(p-1)^{2}} \sum_{\substack{\chi \in \mathcal{X} \\ \chi^{i}, \chi^{j} \neq \chi_{0}}}\left|\sum_{r=R+1}^{R+M} \chi^{i}(r)\right|^{4} \times \sum_{\substack{\chi \in \mathcal{X} \\ \chi^{i}, \chi^{j} \neq \chi_{0}}}\left|\sum_{s=S+1}^{S+M} \chi^{j}(s)\right|^{4} .
$$

When $\chi$ runs through $\mathcal{X}$ the power $\chi^{h}$ represents any other character in $\mathcal{X}$ no more that $h$ times. Thus

$$
\sum_{\substack{\chi \in \mathcal{X} \\ \chi^{i}, \chi^{j} \neq \chi_{0}}}\left|\sum_{r=R+1}^{R+M} \chi^{i}(r)\right|^{4} \ll \sum_{\chi \in \mathcal{X}^{*}}\left|\sum_{r=R+1}^{R+M} \chi^{i}(r)\right|^{4}
$$

and similarly for the second double sums over $s$.

Combining the above bounds with inequality (4.3), applying Lemma 2.2, and then using (4.2), we conclude the proof.

Corollary 4.1. For any prime $p$ and any box $\mathfrak{B}$ given by (1.4) and satisfying (1.5) we have,

as $|\mathfrak{B}| \rightarrow \infty$.

$$
I(\mathfrak{B}) \geq \min \left\{p\left(1+O\left(|\mathfrak{B}|^{-1+o(1)} p\right),|\mathfrak{B}| p^{o(1)}\right\}\right.
$$

Proof. Let $\Gamma=\left\{r^{3} / s^{2}:(r, s) \in \mathfrak{B}\right\}$, we recall that

$$
N_{\lambda}(\mathfrak{B})=\left|\left\{(r, s) \in \mathfrak{B}: r^{3} / s^{2}=\lambda\right\}\right| .
$$

Using the Cauchy inequality we derive

$$
\left.|\mathfrak{B}|^{2}=\left(\sum_{\lambda \in \Gamma} N_{\lambda}(\mathfrak{B})\right)\right)^{2} \leq|\Gamma| \sum_{\lambda} N_{\lambda}^{2}(\mathfrak{B}) \leq I(\mathfrak{B}) T_{3,2}(\mathfrak{B}) .
$$

We conclude the proof by estimating $T_{3,2}(\mathfrak{B})$ with Theorem 4.1 .

It is easy to see that the error term of Theorem 4.1 and thus the second term of Corollary 4.1 can be replaced with $|B|^{1+o(1)}$. 


\section{Bound on $N_{\lambda}(\mathfrak{B})$}

It is easy to see that for $\lambda \in \mathbb{F}_{p}^{*}$ the curve $X^{3}=\lambda Y^{2}$ is absolutely irreducible. So general bounds on the number of points on a curve in a given box (see, for example, [14]) immediately imply that

$$
N_{\lambda}(\mathfrak{B})=\frac{|\mathfrak{B}|}{p}+O\left(p^{1 / 2}(\log p)^{2}\right),
$$

which gives a trivial upper bound when $|\mathfrak{B}| \ll p^{1 / 2} \log p$.

We are now ready to derive a nontrivial upper bound on $N_{\lambda}(\mathfrak{B})$ for smaller values of $M$.

Lemma 5.1. For any prime $p$, any box $\mathfrak{B}$, given by (1.4) and with $1 \leq|\mathfrak{B}| \leq p^{2 / 9}$, satisfying (1.5) and $\lambda \in \mathbb{F}_{p}^{*}$ we have

$$
N_{\lambda}(\mathfrak{B}) \leq|\mathfrak{B}|^{1 / 6+o(1)}
$$

as $|B| \rightarrow \infty$.

Proof. We have to estimate the number of solutions to

$$
(R+x)^{3} \equiv \lambda(S+y)^{2} \quad(\bmod p),
$$

with $1 \leq x, y \leq M$, which is equivalent to the congruence

$$
x^{3}+3 R x^{2}+3 R^{2} x-\lambda y^{2}-2 \lambda S y \equiv \lambda S^{2}-R^{3} \quad(\bmod p) .
$$

For any $T \leq p^{1 / 4} / M^{1 / 2}$, we can apply Lemma 3.2 to

$$
a_{1}=1, \quad a_{2}=3 R, \quad a_{3}=3 R^{2}, \quad a_{4}=-\lambda, \quad a_{5}=-2 \lambda S
$$

and

$$
T_{1}=T^{4} M^{2}, \quad T_{2}=T_{4}=p /(T M), \quad T_{3}=T_{5}=p / T,
$$

and conclude that there exits $|t| \leq T^{4} M^{2}$ with $\operatorname{gcd}(t, p)=1$ such that

$$
\|3 R t\|_{p} \leq p /(T M), \quad\|\lambda t\|_{p} \leq p /(T M), \quad\left\|3 R^{2} t\right\|_{p} \leq p / T, \quad\|2 \lambda S t\|_{p} \leq p / T .
$$

Thus, by multiplying both sides of the congruence (5.2) by $t$, we can replace the congruence (5.2) with the following equation over $\mathbb{Z}$ :

$$
A_{1} x^{3}+A_{2} x^{2}+A_{3} x+A_{4} y^{2}+A_{5} y+A_{6}=p z,
$$

where

$$
\left|A_{1}\right| \leq T^{4} M^{2}, \quad\left|A_{2}\right|,\left|A_{4}\right| \leq p /(T M), \quad\left|A_{3}\right|,\left|A_{5}\right| \leq p / T, \quad\left|A_{6}\right| \leq p / 2 .
$$

Since, for $0 \leq x, y \leq M$, the left hand side of equation (5.3) is bounded by $T^{4} M^{5}+$ $4 p M / T+p / 2$, it follows that

$$
|z| \ll \frac{T^{4} M^{5}}{p}+\frac{4 M}{T}+1 .
$$

The choice $T \sim p^{1 / 5} / M^{4 / 5}$ leads us to the bound

$$
|z| \ll M^{9 / 5} p^{-1 / 5}+1 \ll 1
$$

provided that $M=|\mathfrak{B}|^{1 / 2} \leq p^{1 / 9}$.

We note that the polynomial $A_{1} X^{3}+A_{2} X^{2}+A_{3} X+A_{4} Y^{2}+A_{5} Y+A_{6}$ on left-hand side of (5.3) is absolutely irreducible. Indeed, it is obtained from $X^{3}-\lambda Y^{2}$ (which is 
an absolutely irreducible polynomial) by a nontrivial modulo $p$ affine transformation. Therefore, for every integer $z$, the polynomial $A_{1} X^{3}+A_{2} X^{2}+A_{3} X+A_{4} Y^{2}+A_{5} Y+$ $A_{6}-p z$ is also absolutely irreducible (as its reduction modulo $p$ is absolutely irreducible modulo $p$ ).

Thus, for each $z$ in the previous range, equation (5.3) corresponds to an absolutely irreducible curve of degree 3 which, by Lemma 3.1 , has at most $M^{1 / 3+o(1)}$ points lying in $[0, M]^{2}$. Therefore, the number of solutions in the original equation is bounded by $M^{1 / 3+o(1)}=|\mathfrak{B}|^{1 / 6+o(1)}$.

The family of curves $E_{r, s}$ with $(r, s)=\left(t^{2}, t^{3}\right), 1 \leq t \leq|\mathfrak{B}|^{1 / 6}$, shows that the exponent $1 / 6$ in the bound of Lemma 5.1 cannot be improved, which means that we cannot obtain a general bound stronger than $N_{\lambda}(\mathfrak{B})=O\left(|\mathfrak{B}|^{1 / 6}\right)$.

Clearly the argument used in the proof of Lemma 5.1 works for large values of $|\mathfrak{B}|$. In particular, for $|\mathfrak{B}|>p^{2 / 9}$, it leads to the bound $N_{\lambda}(\mathfrak{B}) \ll|\mathfrak{B}|^{16 / 15+o(1)} p^{-1 / 5}$ which is nontrivial for $|\mathfrak{B}| \leq p^{6 / 17}$.

However, using a modification of this argument we can obtain a stronger bound which is nontrivial for $p^{2 / 9}<|\mathfrak{B}| \leq p^{2 / 5}$ :

Lemma 5.2. For any prime $p$, any box $\mathfrak{B}$, given by (1.4) with $p^{2 / 9}<|\mathfrak{B}| \leq p^{2 / 5}$, satisfying (1.5) and $\lambda \in \mathbb{F}_{p}^{*}$ we have

$$
N_{\lambda}(\mathfrak{B}) \leq|\mathfrak{B}|^{11 / 12+o(1)} p^{-1 / 6}
$$

as $|\mathfrak{B}| \rightarrow \infty$.

Proof. Let $K=\left\lfloor p^{1 / 6} / M^{1 / 2}\right\rfloor$ and observe that we have $1 \leq K \leq M$ when $p^{2 / 9}<$ $|\mathfrak{B}|=M^{2}$. Also observe that one could cover $\mathfrak{B}$ with $J=O(M / K)$ rectangles of the form $\left[R_{j}+1, R_{j}+K\right] \times[S+1, S+M], j=1, \ldots, J$. Then, the equation in each rectangle can be written as

$$
x^{3}+3 R_{j} x^{2}+3 R_{j}^{2} x-\lambda y^{2}-2 \lambda S y \equiv \lambda S^{2}-R_{j}^{3} \quad(\bmod p)
$$

with $1 \leq x \leq K$ and $1 \leq y \leq M$.

To estimate the number of solutions to $(5.4)$, we set

$$
T_{1}=p^{1 / 2} M^{3 / 2}, \quad T_{2}=p^{2 / 3} M, \quad T_{3}=p^{5 / 6} M^{1 / 2}, \quad T_{4}=p / M^{2}, \quad T_{5}=p / M .
$$

and apply, once more, Lemma 3.2 where $a_{i}$ are the coefficients of $x, y$ in (5.4). Hence, as in the proof of Lemma 5.1 , we obtain an equivalent equation over $\mathbb{Z}$ :

$$
A_{1} x^{3}+A_{2} x^{2}+A_{3} x+A_{4} y^{2}+A_{5} y+A_{6}=p z,
$$

where $\left|A_{i}\right| \leq T_{i}$ for $i=1, \ldots, 5$ and $\left|A_{6}\right| \leq p / 2$. The left-hand side of (5.5) is bounded by

$$
\begin{aligned}
& \left|A_{1} K^{3}+A_{2} K^{2}+A_{3} K+A_{4} M^{2}+A_{5} M+A_{6}\right| \\
& \leq p^{1 / 2} M^{3 / 2}\left(\frac{p^{1 / 6}}{M^{1 / 2}}\right)^{3}+p^{2 / 3} M\left(\frac{p^{1 / 6}}{M^{1 / 2}}\right)^{2}+p^{5 / 6} M^{1 / 2} \frac{p^{1 / 6}}{M^{1 / 2}} \\
& \quad+\frac{p}{M^{2}} M^{2}+\frac{p}{M^{2}} M+p / 2 \\
& =5.5 p .
\end{aligned}
$$


Thus, $z$ can take at most 11 values. As we have seen in the proof of Lemma 5.1, the polynomial on the left-hand side of (5.5) is absolutely irreducible. Therefore, Lemma 3.1 implies that, for each value of $z$, equation (5.5) has at most $M^{1 / 3+o(1)}$ solutions. Summing over all rectangles we finally obtain that the original congruence has at most

$$
(M / K) M^{1 / 3+o(1)}=M^{11 / 6+o(1)} p^{-1 / 6}=|\mathfrak{B}|^{11 / 12+o(1)} p^{-1 / 6}
$$

solutions.

Combining (5.1) with Lemmas 5.1 and 5.2, we obtain:

Theorem 5.1. For any prime $p$, box $\mathfrak{B}$ given by (1.4) and satisfying (1.5) and $\lambda \in \mathbb{F}_{p}^{*}$ we have,

$$
N_{\lambda}(\mathfrak{B}) \ll|\mathfrak{B}|^{o(1)} \begin{cases}|\mathfrak{B}|^{1 / 6}, & \text { if }|\mathfrak{B}|<p^{2 / 9}, \\ |\mathfrak{B}|^{11 / 12} p^{-1 / 6}, & \text { if } p^{2 / 9} \leq|\mathfrak{B}|<p^{2 / 5}, \\ p^{1 / 2}, & \text { if } p \leq|\mathfrak{B}|<p^{3 / 2}, \\ |\mathfrak{B}| p^{-1}, & \text { if } p^{3 / 2} \leq|\mathfrak{B}|<p^{2},\end{cases}
$$

as $|\mathfrak{B}| \rightarrow \infty$.

We note that unfortunately in the range $p^{2 / 5} \leq|\mathfrak{B}|<p$ we could not find any nontrivial estimate.

\section{Comments and open problems}

Observe that Theorem 4.1 can be easily extended to coefficients $(r, s)$ that belong to rectangles $[R+1, R+K] \times[S+1, S+L]$ rather than squares (the bound (5.1) also holds for such rectangles).

As we have mentioned the exponent $1 / 6$ in the bound of Lemma 5.1 cannot be improved, however, the range $|\mathfrak{B}| \leq p^{2 / 9}$ can possibly be extended. As the first step towards this, the following question has to be answered:

Problem 6.1. Let $E$ be an elliptic given by a Weierstrass equation

$$
y^{2}+a_{1} x y+a_{3} y=x^{3}+a_{2} x^{2}+a_{4} x+a_{6}, a_{i} \in \mathbb{Z},
$$

such that all the coefficients are $M^{o(1)}$. Is it true that the number of integer points $(x, y) \in[0, M] \times[0, M]$ on $E$ is $M^{o(1)}$ ? boxes.

We refer to $[7,10]$ for some bounds on the number of points on elliptic curves in

As we have noticed in Section 5 we have not found nontrivial bounds on $N_{\lambda}(\mathfrak{B})$ for $p^{2 / 5} \leq|\mathfrak{B}|<p$. It is certainly interesting to close this gap.

Problem 6.2. Is it true that $N_{\lambda}(\mathfrak{B})=o\left(|\mathfrak{B}|^{1 / 2}\right)$ for all $|\mathfrak{B}|=o\left(p^{2}\right)$ ?

Finally, it is also natural to expect that the term $|\mathfrak{B}|^{o(1)}$ can be removed from the result obtained in Corollary 4.1.

Problem 6.3. Is it true that $I(\mathfrak{B}) \gg \min \{p,|\mathfrak{B}|\}$ ? 


\section{Acknowledgment}

The authors are grateful to Moubariz Garaev and Joe Silverman for their comments.

This work started during a very pleasant visit by I. S. to the Universidad Autónoma de Madrid; the support and hospitality of this institution are gratefully acknowledged.

During the preparation of this paper, J.C. was supported by grant MTM 200803880 of MICINN (Spain), I.S. was supported in part by ARC grant DP1092835 (Australia) and by NRF grant CRP2-2007-03 (Singapore), and A.Z. was supported by Departamento de Matemáticas, UAM (Spain).

\section{References}

[1] A. Ayyad, T. Cochrane and Z. Zheng, The congruence $x_{1} x_{2} \equiv x_{3} x_{4}(\bmod p)$, the equation $x_{1} x_{2}=x_{3} x_{4}$ and the mean value of character sums, J. Number Theory 59 (1996), 398-413.

[2] W. D. Banks and I. E. Shparlinski, Sato-Tate, cyclicity, and divisibility statistics on average for elliptic curves of small height, Israel J. Math. 173 (2009), 253-277.

[3] E. Bombieri and J. Pila, The number of integral points on arcs and ovals, Duke Math. J. 59 (1989), 337-357.

[4] J. Cilleruelo and M. Z. Garaev, Concentration of points on two and three dimensional modular hyperbolas and applications, Geom. Funct. Anal. 21 (2011), 892-904.

[5] J. Cilleruelo, M. Z. Garaev, A. Ostafe and I.E. Shparlinski, On the concentration of points of polynomial maps and applications, Math. Z., to appear.

[6] T. Cochrane and S. Sih, The congruence $x_{1} x_{2} \equiv x_{3} x_{4}(\bmod p)$ and mean values of character sums, J. Number Theory 130 (2010), 767-785.

[7] J. S. Ellenberg and A. Venkatesh, Reflection principles and bounds for class group torsion, Int. Math. Res. Not. 2007 (2007), article ID rnm002, 1-18.

[8] É. Fouvry and M. R. Murty, On the distribution of supersingular primes, Canad. J. Math. 48 (1996), 81-104.

[9] M. Z. Garaev and V. Garcia, The equation $x_{1} x_{2}=x_{3} x_{4}+\lambda$ in fields of prime order and applications, J. Number Theory 128 (2008), 2520-2537.

[10] H. A. Helfgott and A. Venkatesh, Integral points on elliptic curves and 3-torsion in class groups, J. Amer. Math. Soc. 19 (2006), 527-550.

[11] H. Iwaniec and E. Kowalski, Analytic number theory, Amer. Math. Soc., Providence, RI, 2004.

[12] H. W. Lenstra, Factoring integers with elliptic curves, Ann. of Math. 126 (1987), 649-673.

[13] J. H. Silverman, The arithmetic of elliptic curves, Springer-Verlag, Berlin, 2009.

[14] M. Vâjâitu and A. Zaharescu, Distribution of values of rational maps on the $\mathbb{F}_{p}$-points on an affine curve, Monatsh. Math. 136 (2002), 81-86.

[15] A. Zumalacárregui, Concentration of points on modular quadratic forms, Internat. J. Number Theory 7 (2011), 1835-1839.

Instituto de Ciencias Matemáticas (CSIC-UAM-UC3M-UCM) And Departamento De Matemáticas, Universidad Autónoma de Madrid, Madrid 28049, España

E-mail address: franciscojavier.cilleruelo@uam.es

Department of Computing, Macquarie University, Sydney, NSW 2109, Australia

E-mail address: igor.shparlinski@mq.edu.au

Instituto de Ciencias Matemáticas (CSIC-UAM-UC3M-UCM) and Departamento DE Matemáticas, Universidad Autónoma de Madrid, Madrid 28049, España

E-mail address: ana.zumalacarregui@uam.es 
